Chinese Journal of Organic Chemistry

\title{
禾谷镰刀菌中的新镰刀菌素
}

\author{
林月婷 ${ }^{\dagger}, a$ 杨 谦 ${ }^{\dagger}, b$ 王婉秋 $c$ 李秋菊 $c$ 汤志军 $b$ \\ 唐威华 $c$ 陶疆*,a 刘文*,b,d \\ $\left({ }^{a}\right.$ 上海交通大学医学院附属第九人民医院口腔综合科 上海交通大学口腔医学院 国家口腔医学中心 \\ 国家口腔疾病临床医学中心 上海市口腔医学重点实验室 上海 200011) \\ ( $b$ 中国科学院上海有机化学研究所 生命有机化学国家重点实验室 上海 200032) \\ ( ${ }^{c}$ 中国科学院植物生理生态研究所 分子植物科学创新中心 上海 200032) \\ ( ${ }^{d}$ 中国科学院上海有机化学研究所 湖州生物制造中心 浙江湖州 313000)
}

\begin{abstract}
摘要 镰刀菌素是由植物病原真菌产生的一类聚酮-非核糖体肽真菌毒素. 其核心骨架的构筑依赖于一个不寻常的聚 酮-非核糖体肽杂合酶. 报道了禾谷镰刀菌中两个不同类型的新镰刀菌素，镰刀菌素 X1 (1)和镰刀菌素 Y (2). 其中，化 合物 1 为聚酮一非核糖体肽杂合化合物，化合物 2 属于聚酮类化合物. 通过基因敲除、高分辨质谱分析证实它们是由同 一个生物合成基因簇产生的主线产物和旁支产物. 该结果为后期进一步研究镰刀菌素生物合成提供了研究基础，也进 一步为该病原真菌侵染禾谷类作物的机制研究提供了化学多样性.
\end{abstract}

关键词 禾谷镰刀菌; 镰刀菌素; 生物合成; 基因敲除

\section{New Fusarins from Fusarium graminearum}

$\begin{array}{cccc}\text { Lin, Yueting }^{\dagger, a} & \text { Yang, Qian }^{\dagger, b} & \text { Wang, Wanqiu }^{c} & \text { Li, Qiuju }^{c}\end{array} \quad$ Tang, Zhijun $^{b}$

$\left({ }^{a}\right.$ Department of General Dentistry, Shanghai Ninth People's Hospital, Shanghai Jiao Tong University School of Medicine, College of Stomatology, Shanghai Jiao Tong University, National Center for Stomatology; National Clinical Research Center for Oral Diseases; Shanghai Key Laboratory of Stomatology, Shanghai 200011)

( ${ }^{b}$ State Key Laboratory of Bioorganic and Natural Products Chemistry, Shanghai Institute of Organic Chemistry, Chinese Academy of Sciences, Shanghai 200032)

( ${ }^{c}$ CAS Center for Excellence in Molecular Plant Sciences, Institute of Plant Physiology and Ecology,

Chinese Academy of Sciences, Shanghai 200032)

( ${ }^{d}$ Huzhou Center of Bio-synthetic Innovation, Shanghai Institute of Organic Chemistry, Chinese Academy of Sciences, Huzhou, Zhejiang 313000)

\begin{abstract}
Fusarins as a group of polyketide-nonribosomal peptide hybrid mycotoxins, are produced by the plant pathogenic fungus Fusarium. The building of core framework depends on an unusual iterative type I polyketide synthase (PKS) fused to a non-ribosomal peptide synthetase (NRPS). Two new compounds, fusarins X1 (1) and Y (2), were isolated from Fusarium graminearum. Fusarin X1 (1) possesses a PKS-NRPS hybrid framework, and fusarin Y (2) is a polyketide. It is demonstrate that they are the main and shunt products in fusarins biosynthetic pathway, respectively, by gene inactivation and HRESIMS analyses. These findings not only lay the foundation for understanding of the fusarins biosynthetic logic, but also provide chemical diversity to further explore the concerning infect mechanism.

Keywords Fusarium graminearum; fusarins; biosynthesis; gene knockout
\end{abstract}

\footnotetext{
* Corresponding authors. E-mail: taojiang_doctor@hotmail.com; wliu@mail.sioc.ac.cn

Received June 25, 2021; revised July 9, 2021; published online July 23, 2021.

Project supported by the National Natural Science Foundation of China (Nos. 81974495, 32030002, 21977109, 21621002), and the National Key Research and Development Program of China (Nos. 2018YFA090100, 2019YFA0905400).

国家自然科学基金(Nos. 81974495, 32030002, 21977109, 21621002)、科技部重大专项(Nos. 2018YFA090100, 2019YFA0905400)资助项目.

$\dagger$ 共同第一作者(These authors contributed equally to this work).
} 
小麦赤霉病(Fusarium head blight, FHB)是影响禾谷 类作物生长的一种毁灭性真菌病害 ${ }^{[1]}$. 禾谷镰刀菌 (Fusarium graminearum) 是引起禾谷类作物感染小麦赤 霉病的主要病原真菌. 得益于禾谷镰刀菌全基因组数据 的公布 ${ }^{[2]}$, 国内外多个研究组对禾谷镰刀菌中与致病相 关的基因进行了研究. 前期研究结果表明禾谷镰刀菌在 侵染谷物类作物时, 能够产生一系列真菌毒素, 如呕吐 毒素脱氧雪腐镰刀霉烯醇 $(\mathrm{DON})^{[3]}$ 、玉米赤霉烯酮 (zearalenone) ${ }^{[4]}$ 以及镰刀菌素(fusarins) $)^{[5]}$ 等. 其中, 镰刀 菌素是一类聚酮-非核糖体肽(PKs-NRPs)杂合的天然产 物, 前期的生物合成研究表明该类化合物由一个不寻常 的 PKS-NRPS 合成酶负责其核心骨架的构筑，随后通过 多步后修饰反应引起其结构多样性 ${ }^{[6]}$. 我们在禾谷镰刀 菌的液体发酵培养物中发现了两个新的镰刀菌素(图 1), 利用生物信息学分析、结合基因敲除、LC-MS 分析证实 了它们是由同一个生物合成基因簇负责产生的主线产 物和旁支产物(shunt product). 该结果为后期进一步研 究镰刀菌素生物合成提供了研究基础, 也进一步为该病 原真菌侵染禾谷类作物的机制研究提供了化学多样性.

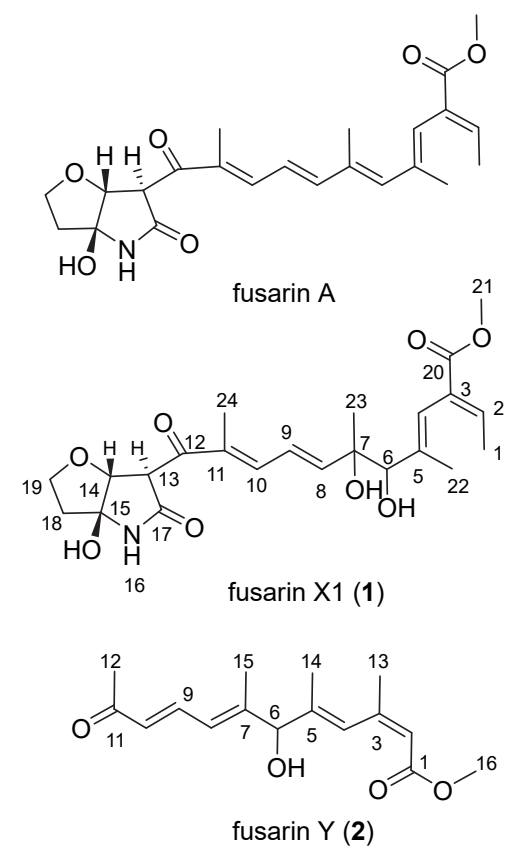

图 1 化合物 fusarins A, X1 (1) 和 Y (2)的化学结构

Figure 1 Chemical structures of fusarins A, X1 (1) and Y (2)

\section{1 结果与讨论}

\section{1 新镰刀菌素的结构鉴定}

镰刀菌素 X1 (1), 淡黄色油状物. 通过高分辨质谱 (HRESIMS) $\mathrm{m} / \mathrm{z} 450.2123[\mathrm{M}+\mathrm{H}]^{+}$(calcd 450.2122)推 测其分子式为 $\mathrm{C}_{23} \mathrm{H}_{31} \mathrm{NO}_{8}$, 含有 9 个不饱和度. 在 ${ }^{13} \mathrm{C}$ NMR 及其 DEPT (distortionless enhancement by polari- zation transfer)谱图中观测到了 23 个碳信号, 包括 5 个 甲基( 1 个甲氧基)、 2 个亚甲基、 8 个次甲基以及 8 个季 碳. 在 ${ }^{1} \mathrm{H} \mathrm{NMR}$ 谱图中存在明显的烯氢、甲氧基以及甲 基信号(表 1). 根据 ${ }^{1} \mathrm{H}-{ }^{1} \mathrm{H}$ COSY (correlation spectroscopy)谱图, 我们建立了 4 个直接相连的片段: C1-C2、 C8-C9-C10、C13-C14 以及 C18-C19. 同时，结合 HMBC (heteronuclear multiple bond correlation)中关键的相关信 号: H-16 $(\mathrm{NH})$ 与 C-13 $\left(\delta_{\mathrm{C}} 56.6\right) 、 \mathrm{C}-14\left(\delta_{\mathrm{C}} 86.1\right) 、 \mathrm{C}-15\left(\delta_{\mathrm{C}}\right.$ 94.1)、 C-17 $\left(\delta_{\mathrm{C}} 169.9\right)$ 相关, $\mathrm{H}_{2}-18$ 与 $\mathrm{C}-14\left(\delta_{\mathrm{C}} 86.1\right)$ 相关, $\mathrm{H}_{2}-19$ 与 $\mathrm{C}-14\left(\delta_{\mathrm{C}} 86.1\right) 、 \mathrm{C}-15\left(\delta_{\mathrm{C}} 94.1\right)$ 相关, $\mathrm{H}-6$ 与 $\mathrm{C}-7$ $\left(\delta_{\mathrm{C}} 75.6\right) 、 \mathrm{C}-23\left(\delta_{\mathrm{C}} 24.5\right)$ 相关, $\mathrm{H}-1$ 与 $\mathrm{C}-3\left(\delta_{\mathrm{C}} 130.3\right)$ 相关, $\mathrm{H}-2$ 与 $\mathrm{C}-20\left(\delta_{\mathrm{C}} 167.0\right)$ 相关, $\mathrm{H}_{3}-23$ 与 C-6 $\left(\delta_{\mathrm{C}} 81.9\right)$ 相关, 以及 $\mathrm{H}_{3}-24$ 与 $\mathrm{C}-11\left(\delta_{\mathrm{C}} 133.8\right) 、 \mathrm{C}-12\left(\delta_{\mathrm{C}} 197.6\right)$ 相关, 确 定了其平面结构(图 2). 随后, 定义 $\mathrm{H}-13$ 为 $\alpha$ 朝向, 通过 NOESY 谱图中 $\mathrm{H}_{3}-1 / \mathrm{H}_{3}-22, \mathrm{H}-2 / \mathrm{H}_{3}-21, \mathrm{H}-10 / \mathrm{H}-8$ 以及 $\mathrm{H}-9 / \mathrm{H}_{3}-24$ 的相关信号, 结合 ${ }^{3} J_{\mathrm{H} 8 / \mathrm{H} 9}=15.1 \mathrm{~Hz}$ 确定 $\Delta^{2}, \Delta^{4}$, $\Delta^{8}$ 和 $\Delta^{10}$ 均为 $E$ 构型. $16-\mathrm{NH} / \mathrm{H}-18 \mathrm{a}, \mathrm{H}-13 / \mathrm{H}-10$ 的相关信 号确定了 $\mathrm{H}-14$ 和 HO-15 为 $\beta$ 朝向. 根据 NOESY (nuclear overhauser effect spectroscopy) 谱图中 H-8/H-6/ $\mathrm{H}-23, \mathrm{H}-8 / \mathrm{H}-22$, 结合文献中 fusarin $\mathrm{G} 1^{[7]}$ 的相关信号,
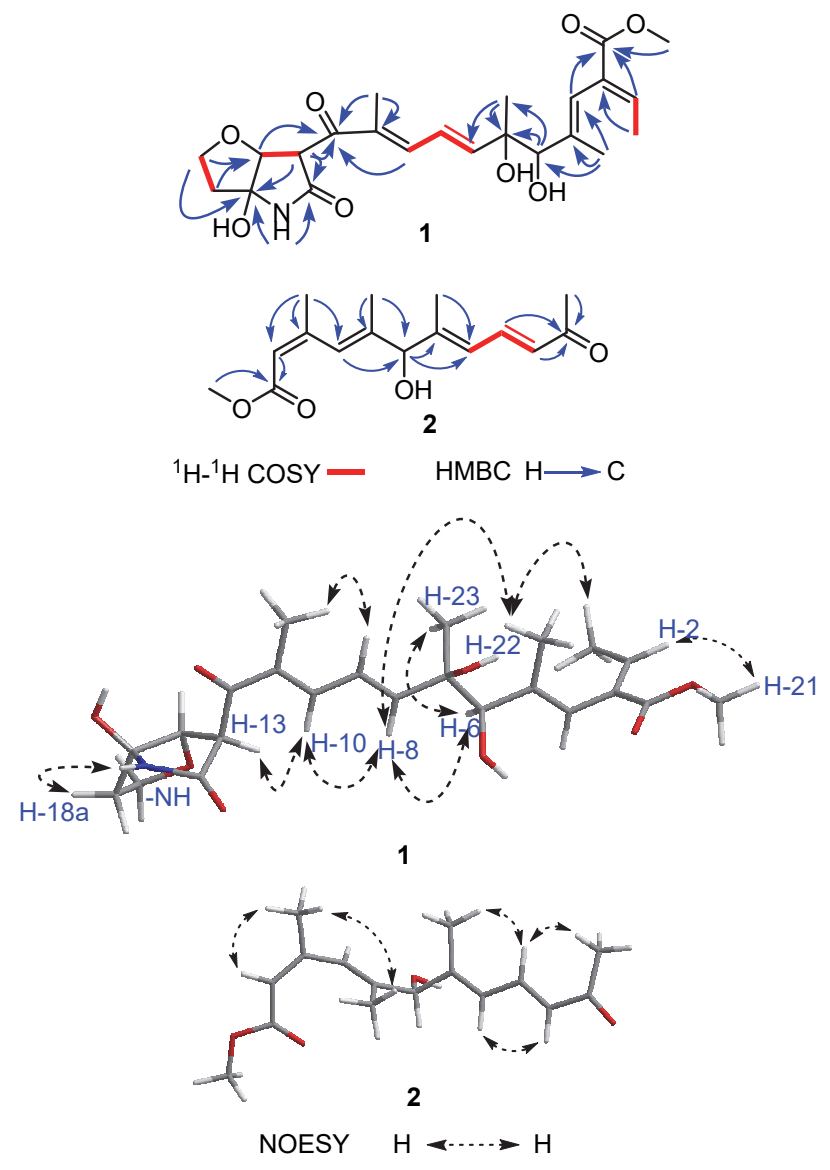

图 2 化合物 $\mathbf{1} \sim 2$ 的 $2 \mathrm{D} \mathrm{NMR}$ 相关图

Figure 2 Key 2D NMR correlations of compounds $\mathbf{1} \sim \mathbf{2}$ 
表 1 Fusarin X1 (1) 和 Fusarin Y (2)在 actone- $d_{6}$ 中测得的 NMR 数据

Table 1 NMR data of fusarins X1 (1) and Y (2) in actone- $d_{6}$

\begin{tabular}{|c|c|c|c|c|}
\hline \multirow{2}{*}{ No. } & \multicolumn{2}{|c|}{ Fusarin X1 (1) } & \multicolumn{2}{|c|}{ Fusarin Y (2) } \\
\hline & $\delta_{\mathrm{H}}(\mathrm{mult}, J / \mathrm{Hz})$ & $\delta_{\mathrm{C}}$, type & $\delta_{\mathrm{H}}(\mathrm{mult}, J / \mathrm{Hz})$ & $\delta_{\mathrm{C}}$, type \\
\hline 1 & $1.74(\mathrm{dd}, 7.2,1.4)$ & $15.0 \mathrm{~s}$ & & $167.8 \mathrm{~s}$ \\
\hline 2 & $6.85 \sim 6.90(\mathrm{~m})$ & $138.8 \mathrm{~d}$ & $6.88($ br s) & $139.7 \mathrm{~d}$ \\
\hline 3 & & $130.3 \mathrm{~s}$ & & $140.0 \mathrm{~s}$ \\
\hline 4 & $6.00 \sim 6.03(\mathrm{~m})$ & $121.1 \mathrm{~d}$ & $6.16 \sim 6.18(\mathrm{~m})$ & $120.8 \mathrm{~d}$ \\
\hline 5 & & $141.0 \mathrm{~s}$ & & $141.4 \mathrm{~s}$ \\
\hline 6 & $4.16(\mathrm{~d}, 2.9)$ & $81.9 \mathrm{~d}$ & $4.65(\mathrm{~s})$ & $80.9 \mathrm{~d}$ \\
\hline 7 & & $75.6 \mathrm{~s}$ & & $150.4 \mathrm{~s}$ \\
\hline 8 & $6.67(\mathrm{~d}, 15.1)$ & $151.3 \mathrm{~d}$ & $6.47 \sim 6.50(\mathrm{~m})$ & $124.1 \mathrm{~d}$ \\
\hline 9 & $6.85 \sim 6.90(\mathrm{~m})$ & $123.7 \mathrm{~d}$ & $7.53(\mathrm{dd}, 15.5,11.5)$ & $139.5 \mathrm{~d}$ \\
\hline 10 & $7.58(\mathrm{dd}, 11.1,1.2)$ & $144.4 \mathrm{~d}$ & $6.15(\mathrm{~d}, 15.5)$ & $131.0 \mathrm{~d}$ \\
\hline 11 & & $133.8 \mathrm{~s}$ & & $198.3 \mathrm{~s}$ \\
\hline 12 & & $197.6 \mathrm{~s}$ & $2.25(\mathrm{~s}, 3 \mathrm{H})$ & $27.3 \mathrm{q}$ \\
\hline 13 & $4.34(\mathrm{~d}, 1.1)$ & $56.6 \mathrm{~d}$ & $1.88(\mathrm{~d}, 1.3)$ & $15.7 \mathrm{q}$ \\
\hline 14 & $4.24(\mathrm{~d}, 1.0)$ & $86.1 \mathrm{~d}$ & $1.38(\mathrm{~d}, 1.3)$ & $13.8 \mathrm{q}$ \\
\hline 15 & & $94.1 \mathrm{~s}$ & $1.88(\mathrm{~d}, 1.3)$ & $13.8 \mathrm{q}$ \\
\hline 16 & $7.97(\mathrm{~s}, \mathrm{NH})$ & & $3.68(\mathrm{~s}, 3 \mathrm{H})$ & $51.9 \mathrm{q}$ \\
\hline 17 & & $169.9 \mathrm{~s}$ & & \\
\hline 18 & $\begin{array}{l}2.26 \sim 2.28(\mathrm{~m}, \\
\text { overlap, } \mathrm{H}-18 \mathrm{a}) \\
2.26 \sim 2.28(\mathrm{~m}, \\
\text { overlap, } \mathrm{H}-18 \mathrm{~b})\end{array}$ & $38.1 \mathrm{t}$ & & \\
\hline 19 & $\begin{array}{l}4.03 \sim 4.05(\mathrm{~m}) \\
3.93 \sim 3.96(\mathrm{~m})\end{array}$ & $67.7 \mathrm{t}$ & & \\
\hline 20 & & $167.0 \mathrm{~s}$ & & \\
\hline 21 & $3.69(\mathrm{~s})$ & $51.0 \mathrm{q}$ & & \\
\hline 22 & $1.59(\mathrm{~d}, 1.4)$ & $14.8 \mathrm{q}$ & & \\
\hline 23 & $1.37(\mathrm{~s})$ & $24.5 \mathrm{q}$ & & \\
\hline 24 & $1.91(\mathrm{~d}, 1.2)$ & $10.7 \mathrm{q}$ & & \\
\hline
\end{tabular}

推测化合物 1 中的 HO-6 和 HO-7 与 fusarin G1 有相同构 型. 镰刀菌素 Y (2), 淡黄色油状物. 根据高分辨质谱 $(+)$-HRESIMS, $[\mathrm{M}+\mathrm{H}]^{+} \mathrm{m} / \mathrm{z} 279.1592$ (calcd 279.1951) 断其分子式为 $\mathrm{C}_{16} \mathrm{H}_{22} \mathrm{O}_{4}$, 不饱和度为 $6 .{ }^{1} \mathrm{H}$ NMR 显示了

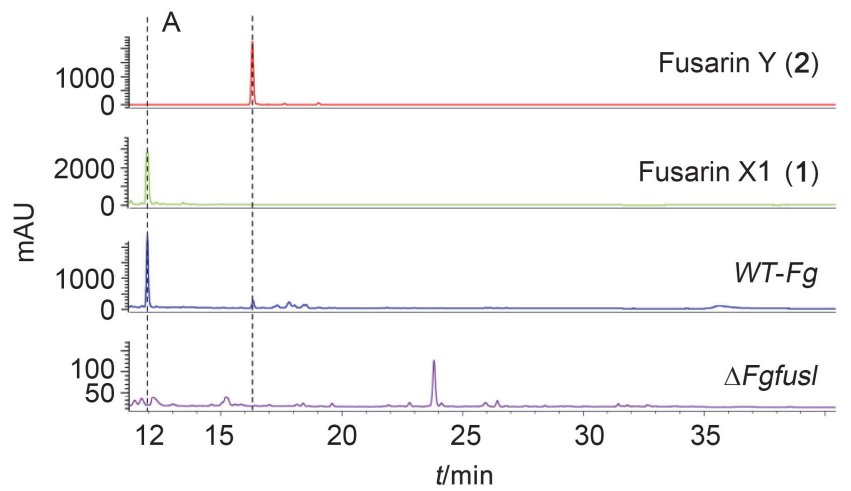

5 个甲基(包括 1 个甲氧基)以及 5 个烯氢信号. ${ }^{13} \mathrm{C} \mathrm{NMR}$ 及其 DEPT 谱图显示了 16 个碳信号, 包括 1 个羰基碳、 1 个酯基碳、 8 个 $\mathrm{sp}^{2}$ 杂化的碳，1 个甲氧基碳和 4 个甲 基碳(表 1). 根据以上信息初步推测该化合物为聚酮类. 通过 ${ }^{1} \mathrm{H}-{ }^{1} \mathrm{H}$ COSY 谱图确定了 C8-C9-C10 的直接相连, 结合关键的 HMBC 信号: $\mathrm{H}_{3}-16$ 与 $\mathrm{C}-1$ 相关, $\mathrm{H}-2$ 与 $\mathrm{C}-1$ 相关, H-6 与 C-7、C-8 相关, $\mathrm{H}_{3}-13$ 与 C-2、C-3、C-4 相 关, $\mathrm{H}_{3}-14$ 与 C-5、C-6 相关, $\mathrm{H}_{3}-12$ 与 C-11 相关. 到此, 化 合物 2 的平面结构被确定. 结合 ${ }^{3} J_{\mathrm{H} 9 / \mathrm{H} 10}=15.5 \mathrm{~Hz}$ 以及 NOESY 谱图中 $\mathrm{H}-2 / \mathrm{H}_{3}-13 / \mathrm{H}_{3}-14, \mathrm{H}-9 / \mathrm{H}_{3}-15, \mathrm{H}-8 / \mathrm{H}-10$ 相 关信号确定 $\Delta^{2}$ 为 $Z$ 构型, $\Delta^{4}, \Delta^{7}$ 和 $\Delta^{9}$ 为 $E$ 构型(图 2).

\section{$1.2 \Delta$ Fgfus1 敲除株的构建及产物分析}

在结构上, 化合物 $\mathbf{1}$ 与 fusarin A (FA) 具有高度的相 似性，区别仅在于化合物 1 中 C6 和 C7 被氧化为二羟基 取代的产物，而 FA 为不饱和双键结构. 同时，化合物 2 与 $\mathbf{1}$ 的侧链结构相似. 基于文献调研，推测化合物 $\mathbf{1}$ 和 $\mathbf{2}$ 可能来源于同一个生物合成基因簇. 为了证实这个观 点, 我们构建了核心骨架基因 $\Delta F g f u s 1$ 突变株 ${ }^{[8]}$. 以天 然产物 1 和 2 为对照品, 利用高分辨高效液相一质谱联用 (HR-HPLC-MS)技术对野生型菌株和 $\Delta F g$ fus 1 突变株进 行对比发酵产物分析(图 3), 在野生型菌株中检测到化 合物 $1\left(\left[\mathrm{M}+\mathrm{NH}_{3}\right]^{+} \mathrm{m} / z\right.$ 467.2390) 和 $2(m / z$ 279.1592, [M $\left.-\mathrm{H}_{2} \mathrm{O}+\mathrm{H}\right]^{+}$261.1487), 然而在 $\Delta F g f u s 1$ 突变株中其对 应的质谱信号均消失了(图 3), 该实验结果证实了我们 先前的推测.

\section{3 化合物 fusarin $X 1$ (1)和 fusarin $Y(2)$ 的生物合成 路径推测}

根据以上实验数据, 推测了对化合物 1 和 $\mathbf{2}$ 的生物 合成路径(图 4). 核心骨架基因 Fgfusl 编码一个非典型 的 PKS-NRPS 杂合酶, 负责催化乙酰辅酶 A (CoA) 和丙
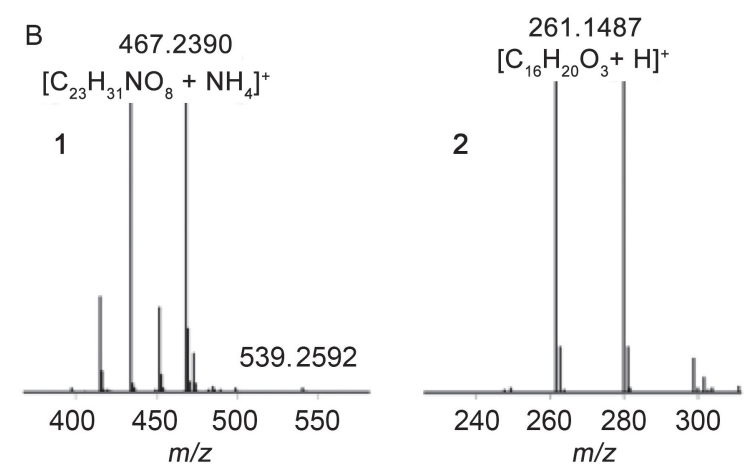

图 3 (A) fusarin X1 (1)、fusarin Y (2)、野生型菌株、 $\Delta F g f u s 1$ 突变株在波长为 $300 \mathrm{~nm}$ 的 HPLC 检测图谱和(B) HRESIMS 检测到 1 和 2 的分子离子峰

Figure 3 (A) HPLC profiles of fusarin X1 (1), fusarin Y (2), and the fermentation extracts of $F$. graminearum (wt and $\Delta F g f u s 1)$ at 330 $\mathrm{nm}$ and (B) HRESIMS-based molecular ion peaks of $\mathbf{1}$ and $\mathbf{2}$ 


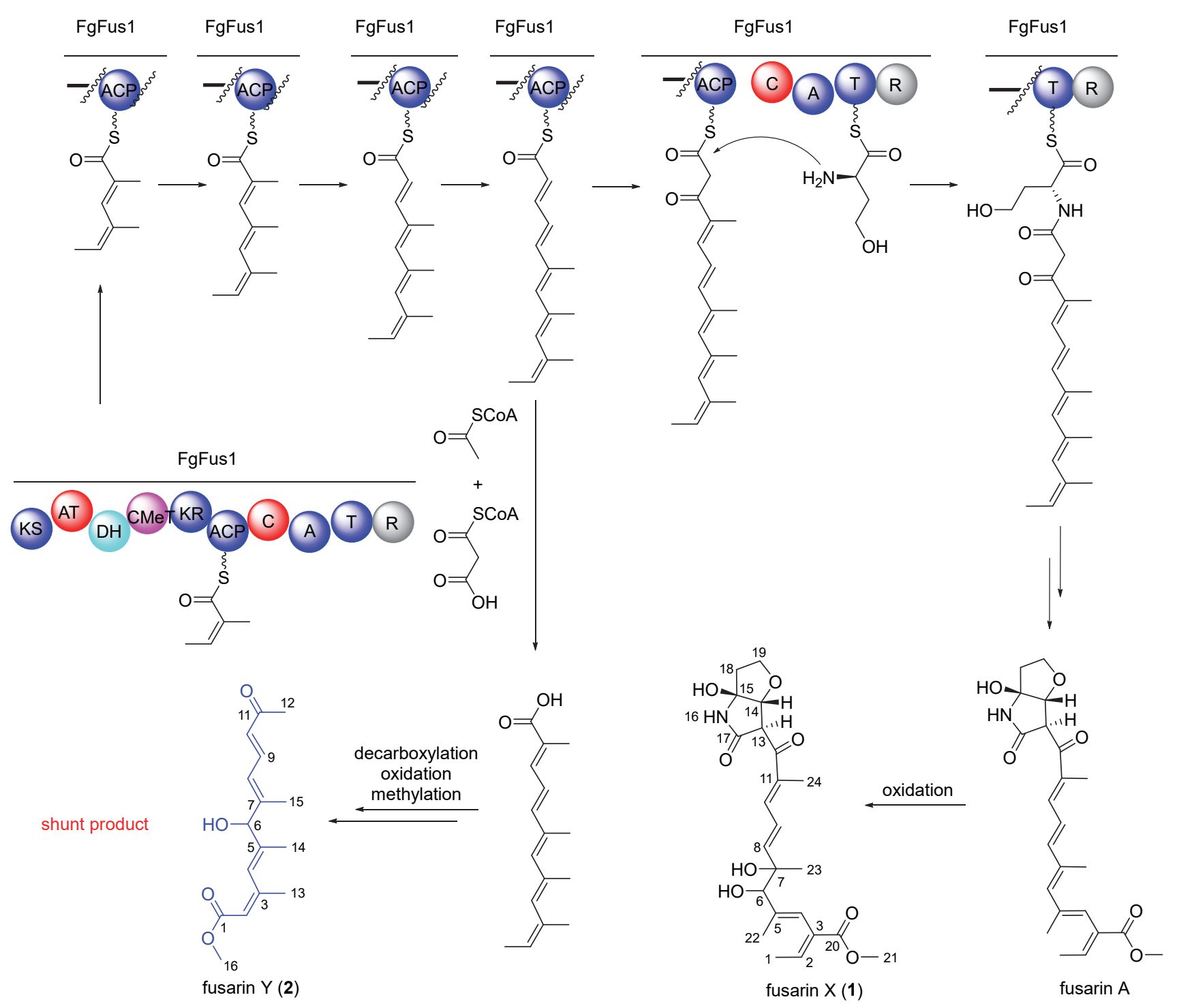

图 4 推测 fusarin X1 (1) 和 fusarin Y (2)的生物合成路径

Figure 4 Proposed biosynthetic pathway of fusarins X1 (1) and Y (2)

二酰辅酶 A (malonyl CoA)发生克莱森缩合反应, 随后 经过装配线 (assembly line)上 DH (dehydratase)、 KR (ketoreductase)和 cMeT (methyltransferase) 功能域进行修 饰, 在 PKS 模块经过 6 次碳链延伸后, 与 NRPS 模块中 挂载的高丝氨酸进行缩合反应, 然后经多步后修饰形成 FA, FA 经过进一步氧化形成化合物 1 . 而化合物 2 可能 是经过 5 轮的二碳单元延伸后下线, 经过脱羧、氧化和 甲基化修饰形成.

\section{4 生物活性测试}

镰刀菌素不仅能够引起动物雌激素紊乱, 同时还具 有肿瘤细胞毒活性. 为了进一步研究镰刀菌素类似物 fusarin X1 (1) 和 fusarin Y (2)的生物活性, 我们测试了它 们对 3 株人类肿瘤细胞的活性(神经胶质瘤细胞 U87、肤 腺癌细胞 PANC-1、皮肤癌细胞 A375). 结果显示, 化合 物 1 显示了中等的肿瘤细胞毒活性, 而化合物 $\mathbf{2}$ 的活性
较弱(图 5). 因此推测, 氮杂环可能是该类化合物发挥生 物活性的重要基团.

\section{2 结论}

禾谷镰刀菌是引起小麦赤霉病最主要的植物病原 真菌. 前人研究表明该菌株的次级代谢产物可能与其侵 染禾谷类作物的机制有着密不可分的关系. 镰刀菌素作 为禾谷镰刀菌产生的一类天然产物, 它们能够引起动物 雌激素紊乱及中毒等现象 ${ }^{[1]}$. 目前已报道的该类化合物 几乎都属于 PKS-NRPS 类, 其结构多样性主要来源于 $\mathrm{N}$-端与侧链修饰程度的差异. 经过对该菌株中次级代谢 产物的进一步挖掘, 分离并鉴定了两个新的镰刀菌素. 基于生物信息学分析，同时利用基因敲除、LC-MS 检测 方法, 证实了它们与其他的镰刀菌素一样，由同一个生 物合成基因簇负责形成. Fusarin X1 (1) 可能是 FA 分子 

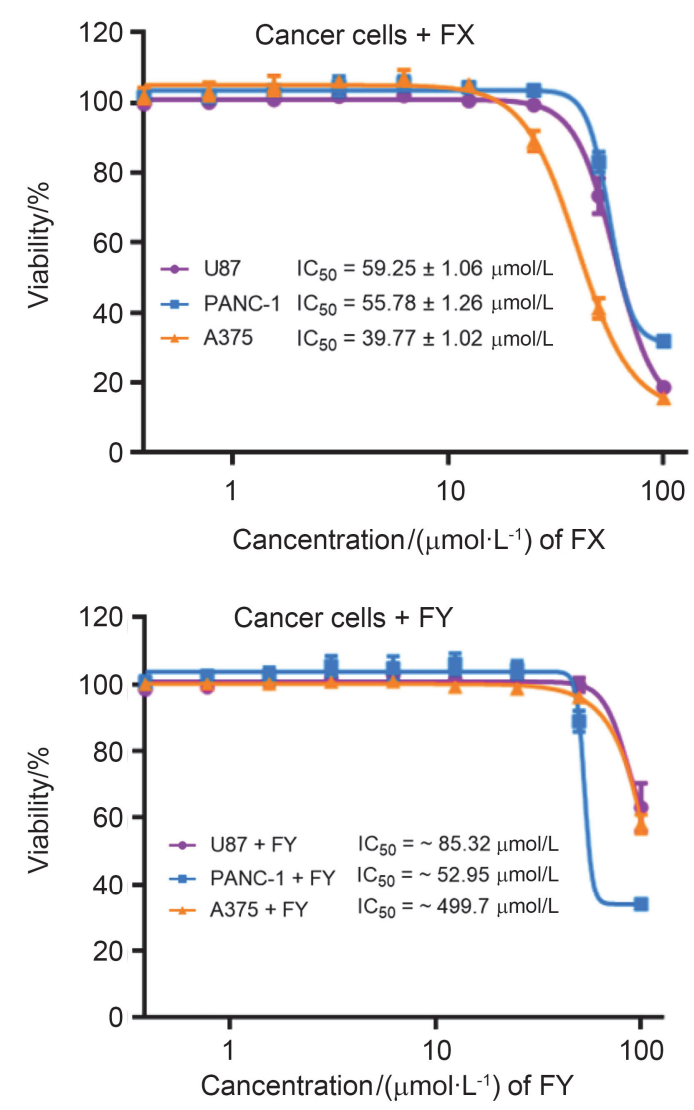

图 5 化合物 $\mathbf{1}$ 和 $\mathbf{2}$ 的生物活性测试结果

Figure 5 Cytotoxic activities of compounds $\mathbf{1}$ and $\mathbf{2}$ against three tumor cell lines

FX: fusarin X1 (1). FY: fusarin Y (2).

经过氧化酶修饰的下游产物, 而 fusarin Y (2)可能是由 PKS 模块进行 5 次延伸后下线, 经小分子水平上的多步 后修饰反应而最终形成的成熟分子. 进一步的 HPLC-MS 分析显示, 野生型菌株中 2 的产量也明显低 于化合物 1, 这与 2 作为旁支产物的结果相符合. 大多 数天然产物生物合成过程常常伴随着不同旁支产物的 产生. 前期 Loesgen 等 ${ }^{[9]}$ 在该菌株中发现了含有 18 个碳 链长的旁支产物, 结合我们的实验结果, 证实该生物合 成基因簇不仅能够合成结构多样的镰刀菌素类天然产 物，而且能够产生不同链长的旁支产物. 同时，体外生 物活性测试表明, 氮杂环可能是该类化合物发挥其生物 功能的关键. 这些发现为后期进一步研究镰刀菌素生物 合成提供了研究基础, 也进一步为该病原真菌侵染禾谷 类作物的机制研究提供了化学多样性.

\section{3 实验部分}

\section{1 仪器与试剂}

Bruker Avance III 500 核磁共振仪: 测 1D 和 2D 核 磁共振谱图; Agilent 6200 series Q-TOF LC-MS: 测 HRESIMS; 正相硅胶：青岛海洋化工厂生产 $(200 \sim 300$
目); 反相硅胶：德国达姆施塔特 Merck 公司生产的 Lichroprep RP-18, 粒径 40 63 $\mu \mathrm{m}$; 凝胶色谱材料: 瑞 典乌普萨拉 Amersham Pharmacia Biotech 公司生产的 Sephadex LH-20 葡聚糖凝胶, 粒径为 $40 \sim 70 \mu \mathrm{m}$; 液相 色谱: Agilent 1260 半制备型 HPLC, 色谱柱 Agilent Zorbax SB-C18 $(9.4 \mathrm{~mm} \times 250 \mathrm{~mm}, 3 \mathrm{~mL} / \mathrm{min})$. 所有 PCR (polymerase chain reaction) 酶等生物试剂购自 Takara 公司, PCR 仪器为 Bio-rad 公司 S1000. 引物合成 由上海铂尚公司完成, 化学试剂购自 Sigma-Aldrich 公 司. 禾谷镰刀菌由中国科学院植物生理生态研究所分子 植物科学卓越创新中心唐威华研究组提供.

\section{2 突变株的构建和菌株的发酵}

Fgfus1 基因的敲除主要采用 Spili-marker 同源重组 技术并进行了相应优化. 通过将需要敲除 Fgfus 1 上游和 下游各 $1 \mathrm{~kb}$ 区域分别与新霉素抗性基因 neo 的前半部分 和后半部分利用融合 PCR 的方式进行拼接，随后将融 合的片段转入野生型禾谷镰狍菌原生质体中, 涂布 TB3 培养基平板后放于 $25{ }^{\circ} \mathrm{C}$ 真菌培养箱内黑暗培养 $3 \mathrm{~d}$, 将 长出的单克隆菌落转接至 $\mathrm{V} 8$ 培养基平板, 继续培养 3 $\mathrm{d}$, 利用 CTAB (cetyltrimethylammonium bromide)法进行 单克隆菌株 DNA 抽提, 并利用内外部引物进行 PCR, 以完成突变体菌株的鉴定. 分别获取三株独立突变体菌 株以完成后续实验. 野生型(WT)和突变菌株都在 V8 固 体培养基上进行 $3 \mathrm{~d}$ 静置培养 (25 ${ }^{\circ} \mathrm{C}$, 避光). 随后挑取 生长旺盛的菌丝接种到 YEPD 培养基(每升含有 $3 \mathrm{~g}$ Yeast Extract, $10 \mathrm{~g}$ Peptone, $20 \mathrm{~g}$ 葡萄糖)，避光条件下， $25{ }^{\circ} \mathrm{C}, 180 \mathrm{r} / \mathrm{min}$ 培养 $7 \mathrm{~d}$. 将菌体过滤, 用乙酸乙酯对 菌液进行萃取, 将乙酸乙酯层低温减压浓缩得到发酵总 产物.

\section{3 化合物的检测和分离}

利用 YEPD 培养基对禾谷镰刀菌进行液体发酵培 养 $(10 \mathrm{~L})$, 发酵液经过离心后, 利用乙酸乙酯萃取, 获得 总浸亳约 $20 \mathrm{~g}$. 利用正向硅胶柱色谱 $\left(\mathrm{CH}_{2} \mathrm{Cl}_{2} / \mathrm{Me}_{2} \mathrm{CO}\right.$, $V: V=1: 0 \sim 0: 1)$ 对总浸亳进行梯度洗脱, 得到 5 个 部分. B 部分 $(4.5 \mathrm{~g})$ 经过反复的正向色谱、反向色谱、凝 胶色谱以及半制备 HPLC 获得化合物 $\mathbf{1}(10.0 \mathrm{mg}), 2$ (3.0 $\mathrm{mg}$ ). 对野生型菌株及突变株发酵液进行检测(A 相加入 $0.1 \%$ 甲酸水, $\mathrm{B}$ 相为加入 $0.1 \%$ 甲酸的乙腈, 均为色谱 级), HPLC 条件: $t=0 \mathrm{~min}, 90 \% \mathrm{~A}, 10 \% \mathrm{~B} ; t=30 \mathrm{~min}, 0 \%$ $\mathrm{A}, 100 \% \mathrm{~B} ; t=35 \mathrm{~min}, 0 \% \mathrm{~A}, 100 \% \mathrm{~B} ; t=40 \mathrm{~min}, 90 \% \mathrm{~A}$, $10 \% \mathrm{~B} ; t=45 \min , 90 \% \mathrm{~A}, 10 \% \mathrm{~B}$. 化合物 1 和 2 的出峰 时间分别为 11.9 和 $16.3 \mathrm{~min}$.

镰刀菌素 X1 (1): 淡黄色油状物. $[\alpha]_{\mathrm{D}}^{20}-4.2$ ( $c 0.1$, $\mathrm{MeOH}) ; \mathrm{UV}(\mathrm{MeOH}) \lambda_{\max }: 290 \mathrm{~nm} ;{ }^{1} \mathrm{H}$ NMR 和 ${ }^{13} \mathrm{C} \mathrm{NMR}$ 数据见 Table 1; IR (KBr) $v_{\text {max }}$ : 3408, 2952, 1701, 1624, 
1436, 1366, 1267, 1136, 1052, $979 \mathrm{~cm}^{-1}$. HRESIMS calcd for $\mathrm{C}_{23} \mathrm{H}_{35} \mathrm{~N}_{2} \mathrm{O}_{8}\left[\mathrm{M}+\mathrm{NH}_{3}\right]^{+}$467.2388, found 467.2390.

镰刀菌素 $\mathrm{Y}(2)$ : 淡黄色油状物. $[\alpha]_{\mathrm{D}}^{20}+5.9$ (c 0.1 , $\mathrm{MeOH}) ; \mathrm{UV}(\mathrm{MeOH}) \lambda_{\max }: 290 \mathrm{~nm} ;{ }^{1} \mathrm{H}$ NMR 和 ${ }^{13} \mathrm{C} \mathrm{NMR}$ 数据见 Table 1; IR (KBr) $v_{\max }$ : 3432, 2950, 2854, 1713, 1664, 1631, 1587, 1435, 1361, 1262, 1192, 1136, 1023, $976 \mathrm{~cm}^{-1}$. positive HRESIMS calcd for $\mathrm{C}_{16} \mathrm{H}_{21} \mathrm{O}_{3}[\mathrm{M}+$ $\mathrm{H}]^{+}$261.1485, found 261.1487.

辅助材料(Supporting Information) 化合物 $\mathbf{1} \sim \mathbf{2}$ 的 HRESIMS, UV, OR, IR, 1D 和 2D NMR 谱图. 这些材料 可以免费从本刊网站(http://sioc-journal.cn/)上下载.

\section{References}

[1] Goswami, R. S.; Kistler, H. C. Mol. Plant Pathol. 2004, 5, 515.

[2] Cuomo, C. A.; Güldener, U.; Xu, J. R.; Trail, F.; Turgeon, B. G.; Di Pietro, A.; Walton, J. D.; Ma, L. J.; Baker, S. E.; Rep, M.; Adam, G.; Antoniw, J.; Baldwin, T.; Calvo, S.; Chang, Y. L.; Decaprio, D.;
Gale, L. R.; Gnerre, S.; Goswami, R. S.; Hammond-Kosack, K. Harris, L. J.; Hilburn, K.; Kennell, J. C.; Kroken, S.; Magnuson, J. K.; Mannhaupt, G.; Mauceli, E.; Mewes, H. W.; Mitterbauer, R.; Muehlbauer, G.; Münsterkötter, M.; Nelson, D.; O'Donnell, K.; Ouellet, T.; Qi, W.; Quesneville, H.; Roncero, M. I.; Seong, K. Y.; Tetko, I. V.; Urban, M.; Waalwijk, C.; Ward, T. J.; Yao, J.; Birren, B. W.; Kistler, H. C. Science 2007, 317, 1400.

[3] Kimura, M.; Tokai, T.; Takahashi-Ando, N.; Ohsato, S.; Fujimura, M. Biosci., Biotechnol., Biochem. 2007, 71, 2105.

[4] Kim, Y.-T.; Lee, Y.-R.; Jin, J.; Han, K.-H.; Kim, H.; Kim, J.-C.; Lee, T.; Yun, S.-H.; Lee, Y.-W. Molecular Microbiology. 2005, 58, 1102.

[5] Wiebe, L. A.; Bjeldanes, L. F. J. Food Sci. 1981, 46, 1424.

[6] Niehaus, E.-M.; Kleigrewe, K.; Wiemann, P.; Studt, L.; Sieber, Christian, M. K.; Connolly, Lanelle, R.; Freitag, M.; Güldener, U.; Tudzynski, B.; Humpf, H.-U. Chem. Biol. 2013, 20, 1055.

[7] Wang, C.-X.; Chen, G.-D.; Feng, C.-C.; He, R.-R.; Qin, S.-Y.; Hu, D.; Chen, H.-R.; Liu, X.-Z.; Yao, X.-S.; Gao, H. Chem. Commun. 2016, 52, 1250.

[8] Yang, Q.; Wang, W.; Lin, Y.; Lin, Y.; Tang, Z.; Wang, J.; Tao, J.; Tang, W.; Liu, W. Org. Biomol. Chem. 2021.

[9] Adpressa, D. A.; Connolly, L. R.; Konkel, Z. M.; Neuhaus, G. F.; Chang, X. L.; Pierce, B. R.; Smith, K. M.; Freitag, M.; Loesgen, S. Fungal Genet. Biol. 2019, 132, 103256. 